\title{
Tumor localization of an anti-TGF-ß antibody and its effects on gliomas
}

\author{
PETRA HÜLPER ${ }^{1}$, WALTER SCHULZ-SCHAEFFER ${ }^{2}$, CHRISTIAN DULLIN $^{3}$, PETER HOFFMANN $^{4}$, \\ JAY HARPER ${ }^{5}$, LESLIE KURTZBERG ${ }^{5}$, SCOTT LONNING ${ }^{5}$, WILFRIED KUGLER ${ }^{1}$, \\ MAX LAKOMEK $^{1}$ and BERNHARD ERDLENBRUCH ${ }^{1,6}$
}

\begin{abstract}
Departments of ${ }^{1}$ Pediatrics I, ${ }^{2}$ Neuropathology and ${ }^{3}$ Diagnostic Radiology, University Medical Center Göttingen, Robert-Koch-Str. 40, D-37099 Göttingen, Germany; ${ }^{4}$ Genzyme Corporation, 675 W Kendall Street, Cambridge, MA 02142;

${ }^{5}$ Cancer and Immunotherapy Research, Genzyme Corporation, 49 New York Avenue, Framingham, MA 01701, USA; ${ }^{6}$ Johannes Wesling Klinikum Minden, Kinderklinik, Hans-Nolte-Str.1, D-32429 Minden, Germany
\end{abstract}

Received July 28, 2010; Accepted September 14, 2010

DOI: 10.3892/ijo_00000823

\begin{abstract}
Even with current standard-of-care therapies, the prognosis for patients with malignant gliomas is very poor and several new treatment modalities for glioblastomas are currently under investigation. Given the role of TGF- $\beta$ in gliomas, anti-TGF- $\beta$ strategies against gliomas are currently being investigated. Biodistribution of intravenously injected AF680-labeled 1D11, a pan-neutralizing TGF- 3 antibody, was monitored in mice bearing either subcutaneous or orthotopic gliomas using in vivo imaging and fluorescence microscopy. AF680-labeled 1D11 entered both the subcutaneous and intracranial tumors and the antibody was detected within the tumor tissue for several days whereas only low fluorescence was found in organs. The effects of 1D11 on subcutaneous versus orthotopic U87MG and GL261 gliomas in immunocompetent C57BL/6J versus immunodeficient CD1-Foxn1nu mice were observed by direct tumor size measurement, H\&E staining and immunohistochemistry. Treatment of immunocompetent mice bearing subcutaneous GL261 tumors with 1D11 resulted in complete remission. In immune deficient mice, the growth of subcutaneous GL261 tumors was increased following treatment with 1D11. Intracranially implanted gliomas in $\mathrm{C} 57 \mathrm{Bl} / 6 \mathrm{~J}$ mice showed no size reduction
\end{abstract}

Correspondence to: Dr Petra Hülper, University Medical Center Göttingen, Dept. of Pediatrics I, Robert-Koch-Str. 40, D-37099 Göttingen, Germany

E-mail: huelper@med.uni-goettingen.de

Abbreviations: TGF- $\beta$, transforming growth factor-beta; $\mathrm{SC}$, subcutaneous; NIR, near infrared; VEGF, vascular endothelial growth factor

Key words: glioblastoma, TGF-ß, 1D11 antibody, immune system, NIR-imaging after 1D11 treatment but there was reduced invasion of the glioma cells into the adjacent normal brain. Together these data demonstrate that TGF- $\beta$ plays different roles in combating the tumor depending on subcutaneous versus orthotopic implantation site.

\section{Introduction}

Glioblastoma multiforme is a highly infiltrative, rapidly growing and highly vascularized tumor. It is characterized by an intrinsic resistance to radio- and chemotherapy, which is responsible for the poor prognosis of patients with highgrade glioma. Despite multimodal treatment, mean survival of the patients remains less than 15 months (1). The improved knowledge of molecular mechanisms in glioma biology has enabled the development of targeted therapies (2). Novel antibodies, antisense oligonucleotides and small molecule inhibitors for the treatment of glioblastomas are under current investigation $(2,3)$ and many others are currently being tested in clinical trials (4-6). In fact, bevacizumab, a neutralizing vascular endothelial growth factor (VEGF) antibody, was recently approved for treatment of glioblastoma multiforme. In view of the elucidation of the high genetic variability in glioblastoma cells, there is mounting evidence that, for successful treatment of glioblastomas, their molecular properties have to be taken into consideration $(7,8)$.

Transforming growth factor-beta (TGF- 3 ) plays a role in the growth and progression of gliomas. Unlike most cancers where TGF- $\beta 1$ is the isoform most commonly overexpressed, TGF- $\beta 2$ is the most abundant isoform upregulated in gliomas. TGF- $\beta$ promotes tumor cell migration and invasion by upregulation of matrix metalloproteinase expression and downregulation of its inhibitors $(9,10)$, whereas inhibition of TGF- $\beta$ reduces migration of glioma cells (11). Gliomas are typically highly vascularized and in these tumors, neovascularization is promoted by TGF- $\beta$-mediated expression of angiogenic factors like VEGF and FGF (12-14). TGF- $\beta$ is a potent immunosuppressant that represses development and antigen presentation of dendritic and other antigen-presenting cells, inhibits natural killer cells and prevents the activation 
and differentiation of cytotoxic $\mathrm{T}$ cells (15-17). Antigenspecific CD8 $\mathrm{T}$ cell effector function is inhibited by the addition of TGF- $\beta$ in vitro (18). Given its role in supporting the growth and invasion of gliomas, TGF- $\beta$ represents a promising target in the treatment of glioblastoma (19).

The pan-neutralizing anti-TGF- $\beta$ antibody, 1D11, has successfully been used in mice to inhibit metastasis in preclinical models of renal cancer (20) and metastatic breast cancer (21). Neutralization of TGF- $\beta$ with 1 D11 enhanced the efficacy of a glioma-associated antigen vaccine resulting in increased survival of mice bearing orthotopic GL261 gliomas and this was attributed to an increase in $\mathrm{CD} 8^{+} \mathrm{T}$ cells and a decrease in regulatory $\mathrm{T}$ cells in the tumor microenvironment (22).

In the present study in vivo near infrared (NIR) imaging, fluorescence microscopy, immunohistochemistry and H\&E staining were performed. Biodistribution studies demonstrate that fluorescently-labeled 1D11 antibody is able to localize to both subcutaneous (SC) and orthotopic gliomas. Furthermore, the effects of 1D11 therapy on SC or orthotopic gliomas in immunocompetent and/or immunodeficient mice were compared to determine the efficacy of neutralizing TGF- $\beta$ in glioma models. Differential effects were observed in these SC models, where 1D11 inhibited the growth of syngeneic tumors and increased apoptosis in these tumors. These effects were not observed in the xenograft model. The effects of 1D11 on orthotopic, syngeneic gliomas were also investigated and although no effect on tumor size was observed, the tumors were less invasive when compared to controls.

\section{Materials and methods}

Antibodies and fluorescent labeling. The pan-neutralizing antiTGF- $\beta$ murine monoclonal IgG1 antibody 1D11 (Genzyme, Corp. Cambridge, MA, USA) was used for treatment of nu/nu and C57BL/6J mice. The antibody binds and neutralizes the active forms of TGF- $\beta 1,2$ and 3 (23). The anti-Shiga toxin murine monoclonal IgG1 antibody 13C4 (Genzyme) was used as an isotype control antibody. The antibody stock solutions were always freshly thawed and diluted with $0.9 \%$ $\mathrm{NaCl}$ to the final concentration before intravenous injection of $100 \mu \mathrm{l}$ per mouse. For imaging analyses, the antibodies were labeled with either Alexa Fluor 594 or Alexa Fluor 680 according to the manufacturer's protocols (Invitrogen, Carlsbad, CA, USA).

Cell culture. U87MG human glioma cells were cultured in MEM-Earle (Biochrom) supplemented with 10\% FCS (Biochrom) and $1 \%$ penicillin/streptomycin $(5000 \mathrm{U} / \mathrm{ml}$, Cambrex, Apen, Germany). GL261 mouse glioma cells were cultured in DMEM (Biochrom) supplemented with 10\% FCS and $1 \%$ penicillin/streptomycin. The cells were incubated at $37^{\circ} \mathrm{C}, 90 \%$ humidity and $5 \% \mathrm{CO}_{2}$, grown in $75 \mathrm{~cm}^{2}$ cell culture flasks (Greiner bio-one, Frickenhausen) and were typically passaged two times a week.

Animal experiments. CD1-Foxn1nu mice and C57BL/6J mice (Charles River, Sulzfeld, Germany) were kept under conventional controlled conditions $\left(22^{\circ} \mathrm{C}, 55 \%\right.$ humidity and $12 \mathrm{~h}$ day-night rhythm) and had free access to either a standard diet (V1534-000, Fa. sniff, Soest, Germany) or an alfalfa-free diet consisting of GLP Nafag feed 890 (Provimi Kliba AG, Kaiseraugst, Switzerland) in preparation for in vivo imaging. The experiments were performed in accordance with the German law on protection of animals and have been approved by the local authorities 'Niedersächsisches Landesamt für Verbraucherschutz und Lebensmittelsicherheit, Oldenburg, Germany'. To reduce hormonal influence on tumor growth, male mice were used. The weight of the animals at the initiation of the studies was between 28 and $32 \mathrm{~g}$.

For the treatment of SC tumors, mice were first anesthetized with an isoflurane/oxygen gas mix and then $1 \times 10^{6}$ human U87MG cells or murine GL261 cells suspended in $100 \mu 1$ phosphate buffered saline were implanted on the right flank of CD1-Foxn1nu mice. Murine GL261 cells were also implanted on the right flank of $\mathrm{C} 57 \mathrm{BL} / 6 \mathrm{~J}$ mice. The tumors were treated intravenously with $4.8 \mathrm{mg} / \mathrm{kg}$ body weight of $1 \mathrm{D} 11$ or $13 \mathrm{C} 4$ at days $7,10,14,17,21$ and 28 after tumor implantation or with $4.8 \mathrm{mg} / \mathrm{kg}$ body weight of AF680 1D11 or AF680 13C4 at days 7, 11, 14, 18 and 25. Tumor size [height (a), length (b) and width (c)] was measured on days $7,10,14,17,21,24,28,31$ and 35 after implantation. The volume was calculated by the formula $4 / 3 \cdot \pi \cdot a / 2 \cdot b / 2 \cdot c / 2$. The animals were sacrificed by narcotic overdose and the tumors were excised.

For the treatment of intracranial tumors, GL261 cells were implanted into the right hemisphere of C57BL/6J mice via stereotactic surgery. The mice were first anesthetized with intraperitoneal injection of ketamine/xylazine $85 \mathrm{mg} \mathrm{kg}^{-1}$ body weight/7 $\mathrm{mg} \mathrm{kg}^{-1}$ body weight and the fur was removed with cold wax strips. After a midline incision, a small hole was drilled into the skull $1 \mathrm{~mm}$ rostral and $2 \mathrm{~mm}$ lateral of the bregma. A volume of $5 \mu 1$ PBS containing $7.5 \times 10^{4}$ tumor cells was injected $3 \mathrm{~mm}$ deep under the dura mater in the brain with a $10 \mu 1$ Hamilton syringe (Hamilton, Bonaduz, Switzerland).

The tumors were treated intravenously with $4.8 \mathrm{mg} / \mathrm{kg}$ body weight with $1 \mathrm{D} 11$ or $13 \mathrm{C} 4$ at days $3,6,10,13$ and 17 after implantation. The animals were sacrificed by narcotic overdose and perfused with $50 \mathrm{ml}$ PBS via cardiac puncture on day 25 after tumor implantation. Excised brains were fixed in $4 \%$ formalin.

Tissue preparation and immunostaining. The dissected tumors were either frozen in 2 -methylbutane $\left(-40^{\circ} \mathrm{C}\right.$; Riedelde-Haën, Seelze, Germany) or fixed in $4 \%$ formalin and embedded in paraffin. Cryosections $(7 \mu \mathrm{m})$ were stained for endothelial cells after 5-min incubation in $100 \%$ ethanol $\left(-20^{\circ} \mathrm{C}\right)$ and blocking in $10 \%$ FCS in PBS for $20 \mathrm{~min}$. Primary antibody was the von Willebrand Factor F8 (1:300) (Dako Cytomation) for $1 \mathrm{~h}$. Secondary antibody was goat anti-rabbit polyclonal antibody Alexa Fluor 594 (1:200) (Invitrogen) for $1 \mathrm{~h}$ followed by $10 \mathrm{~min}$ incubation with DAPI (1:1000). Slices were covered with Fluoromount G (Biozol, Eching, Germany).

Paraffin sections $(1 \mu \mathrm{m})$ were rehydrated and boiled in citrate buffer for antigen retrieval. Endogenous peroxidase was blocked with $100 \%$ methanol, $600 \mu 1 \mathrm{H}_{2} \mathrm{O}_{2}$ at $4^{\circ} \mathrm{C}$, for $20 \mathrm{~min}$. Non-specific protein binding was blocked by incubation in $10 \%$ FCS in PBS for 20 min. Incubation of the first antibody 
was performed overnight at $4^{\circ} \mathrm{C}$ followed by the second antibody for $1 \mathrm{~h}$. Slides were then exposed to peroxidase conjugated biotin $(1: 1000)$ for $1 \mathrm{~h}$ before developing with DAB (Roth, Karlsruhe, Germany). Slices were covered with Depex (Sigma, Deisenhofen, Germany). All incubation steps were performed in a moisture chamber.

All antibodies were diluted in PBS. Rat anti-mouse Mac-3 antibodies (1:300; BD Biosciences) were used for staining macrophages, a mouse anti-human Ki67 antibody MIB-1 (1:500; Dako) was used to determine the proliferation index of tumors and a rabbit anti-human active caspase- 3 antibody, (1:300; BD Biosciences) was used to determine apoptosis. Secondary antibodies were biotinylated sheep anti-mouse polyclonal antibody (1:500; Amersham), biotinylated goat antirat polyclonal antibody (1:200; Amersham), and biotinylated donkey anti-rabbit polyclonal antibody (1:200; Amersham).

In vivo imaging studies. The NIR dye-coupled antibodies AF680 1D11 and AF680 13C4 were administered via tail vein injection at $4.8 \mathrm{mg} / \mathrm{kg}$ body weight. The fluorescent markers were visualized in the time-domain in vivo small animal fluorescence imager Optix ${ }^{\mathrm{TM}}$ (ART, Montreal, QC Canada) (24,25). The excitation laser wavelength was $680 \mathrm{~nm}$ and detection was performed by applying a $700 \mathrm{~nm}$ LP filter. Repeated imaging was performed under anesthesia with an isoflurane/oxygen gas mix. For fluorescence detection a time-correlated single-photon counting system (TCSPC-130) was used. Since the system is time-resolved it is possible to provide fluorescence lifetime in addition to intensity. This lifetime varies between different chromophores and the fluorescence originating from the animal tissue. Thus lifetime measurement can be used to discriminate between fluorescence originating from the dye administered to the animal and auto-fluorescence (26).

Data analysis. Graphs were created with MS Excel, 2003, statistics were obtained with Sigma plot 3.5 software (Systat Software Inc.). Microscopic evaluation was done with a fluorescence and light microscope (Leica DM5000 B). For in vivo imaging fluorescence intensity calculations the program eXplore Optix OptiView was used. Intensity units were normalized for the same excitation power and excitation time (integration time) per dot. Normalized fluorescence intensities are presented in arbitrary units.

\section{Results}

$1 D 11$ specifically reaches experimental gliomas. In gliomas, TGF- $\beta 2$ is significantly upregulated and TGF- $\beta 1$ is often overexpressed as well. In order to determine if the anti-TGF- $\beta$ antibody, 1D11, which binds and neutralizes all three active TGF- $\beta$ isoforms was able to effectively target the tumors in both SC and intracranial settings, 1D11 and 13C4 isotype control antibodies were fluorescently-labeled with Alexa Fluor dyes and in vivo imaging studies were conducted using the NIR-imaging system. Following intravenous administration of fluorescently-labeled antibodies into mice bearing SC tumors, localization of AF680 1D11 in the U87MG tumors could be shown by in vivo fluorescence measurement. Fluorescence was detected in the whole CD1-Foxn1nu mouse $2 \mathrm{~h}$ after intravenous treatment with AF680 labeled-1D11, however fluorescence intensity in the tumor was higher than in the rest of the body (Fig. 1a). There was only low fluorescence of the antibody in organs of CD1-Foxn1nu mice. Sequential measurements of fluorescence intensity in the tumor region showed the presence of 1D11 antibody in the tumor during the treatment period with peaks observed shortly after the intravenous injections. After enrichment in the tumor region the antibody remained in the tumor tissue for at least 4 days. (Fig. 1b). The administration of the same amount of labeled control antibody AF680 13C4 also resulted in increased fluorescence intensity in the mouse with some localization to the tumors (Fig. 1a). However, the enrichment of the AF680 coupled 13C4 antibody in the SC tumors was less marked than with AF680 1D11. Thus, there appears to be a specific enrichment of AF680 1D11 in the tumors represented by the difference between the 13C4 and the 1D11 fluorescence intensity (Fig. 1b).

To determine if the antibody reaches the tumor tissue in SC and orthotopic GL261 tumors in syngeneic C57BL/6J mice, biodistribution of AF680-labeled 1D11 was analyzed in vivo (Fig. $2 \mathrm{a}$ and $\mathrm{b}$ ). Ten minutes after intravenous injection of AF680 1D11, fluorescence intensity was measurable within the SC tumor by in vivo imaging as it was in the SC CD1-Foxn1nu mice (Fig. 2a). Labeled 1D11 is also capable of localizing to orthotopic tumors. Given the difficulties in imaging through the fur, skin and skull of mice bearing orthotopic tumors, the mice were imaged in vivo $10 \mathrm{~min}$ after one single i.v. injection, and then the tumor-bearing brains were excised and imaged ex vivo. On day 5 after implantation no fluorescence intensity was measureable in the tumor region of removed brains (Fig. 2b). Labeled antibody was detectable in the orthotopic gliomas 10 min after i.v. injection of the antibody beginning on day 8. Fluorescence lifetime analysis was conducted to ensure that the fluorescence observed in the gliomas could be attributed to the labeled antibody and not from free dye. The lifetime of 1D11-coupled AF680 is different from that of free AF680 dye in the mouse (Fig. 2c). Moreover, $24 \mathrm{~h}$ after injection free uncoupled AF680 is almost completely eliminated from the mouse, while AF680 1D11 remained in the body.

Finally, fluorescence microscopy of frozen sections was performed on sections of tumors that had grown either SC or orthotopically. Antibodies labeled with Alexa Fluor 594 dye were used for these experiments given that the lower wavelength was more amenable to fluorescence microscopy. Twenty minutes after a single injection of $4.8 \mathrm{mg} / \mathrm{kg} 20$ days post tumor cell injection, the antibody was detectable at the vessel walls and within the tumor tissue of both the SC and orthotopic gliomas (Fig. 2a and b, respectively).

SC glioma treatment with $1 D 11$ leads to tumor shrinkage in immunocompetent mice. Once it was determined that the antibodies could effectively target tumors and could cross the blood-brain/blood-tumor barrier in an orthotopic setting, efficacy studies were carried out to determine if neutralization of TGF- $\beta$ would be efficacious against these tumors. Treatment of C57B1/6J mice bearing subcutaneously implanted GL261 gliomas with $4.8 \mathrm{mg} / \mathrm{kg}$ 1D11 antibody two times a week led to tumor shrinkage with complete remission or tumor 

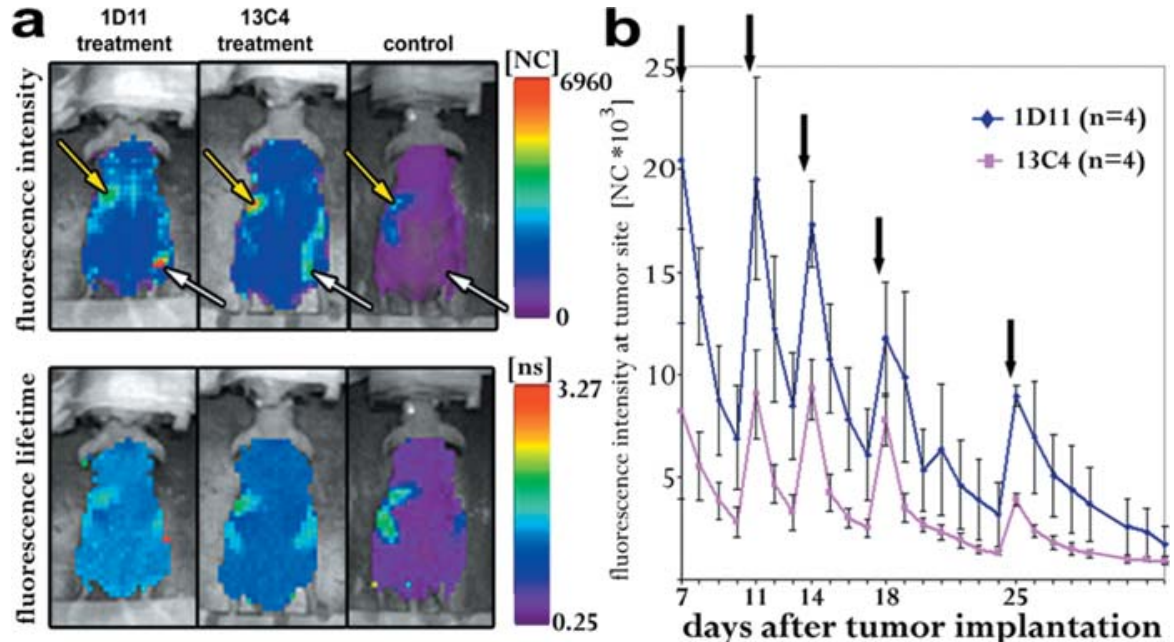

Figure 1. (a) In vivo imaging using AF680 1D11 or AF680 13C4 antibodies. Fluorescence was measured $24 \mathrm{~h}$ after the second i.v. treatment with $4.8 \mathrm{mg} / \mathrm{kg}$ of the fluorescing antibody. The control mouse received no injections. AF680 antibody lifetime is about $1.4 \mathrm{~ns}$. White arrows show the U87MG tumor, yellow arrows show the spleen. (b) Fluorescence intensity in the tumor in mice treated with i.v. AF680 1D11 or AF680 13C4. Daily measurements were started after the first antibody solution was injected 7 days after tumor implantation. Injections were repeated at day 11, 14, 18 and 25 as indicated by black arrows.

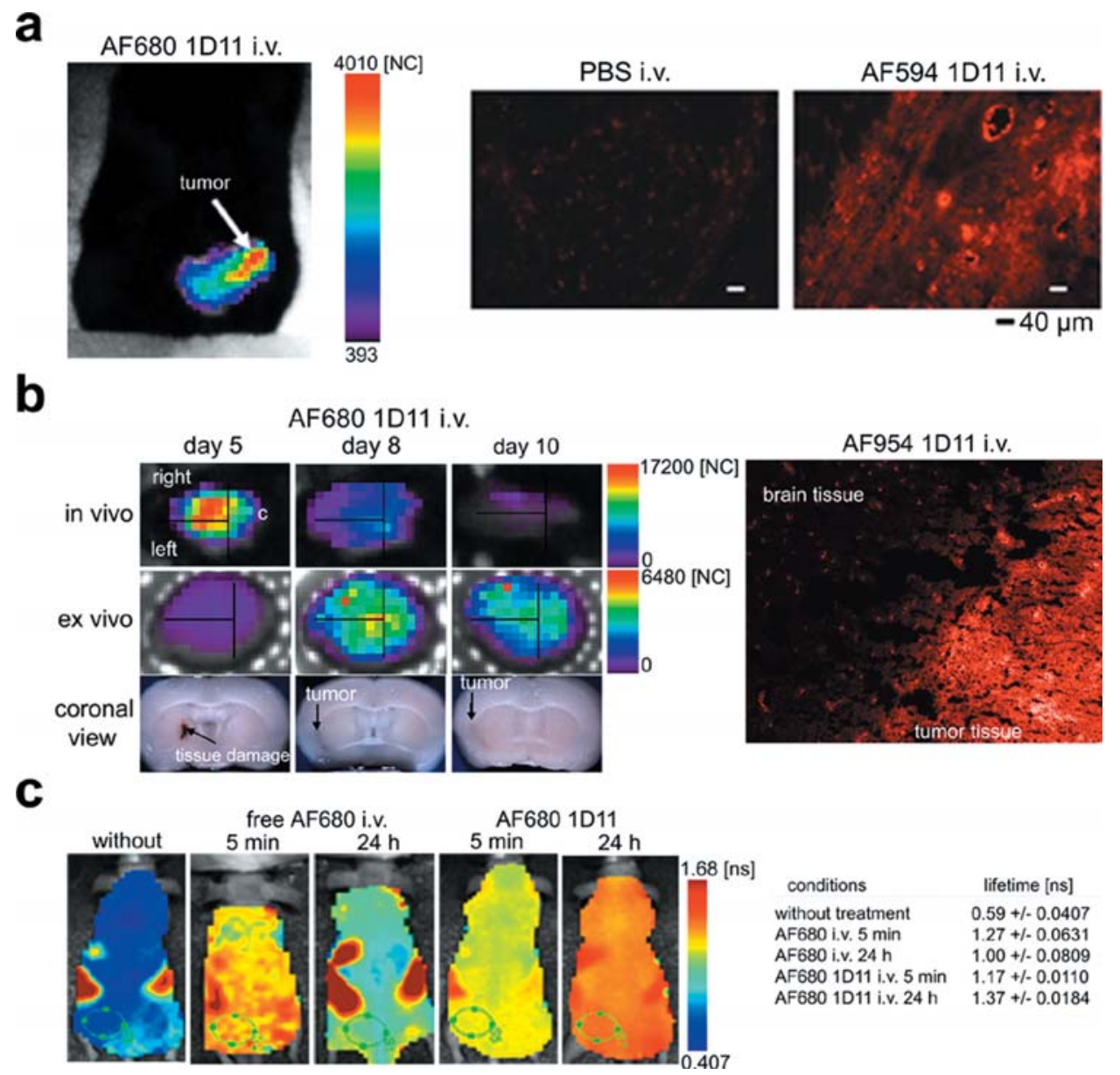

Figure 2. (a) 1D11-coupled AF680 in a subcutaneous tumor of a shaved C57BL/6J mouse 10 min after i.v. injection measured in vivo and fluorescence of i.v. administered 1D11-coupled AF594 in a cryosection of a tumor 25 days after implantation. (b) Fluorescence intensity of 1D11-coupled AF680 in an intracranial glioma of C57BL/6J mice $10 \mathrm{~min}$ after i.v. injection measured in vivo on day 5,8 and 10, respectively. Due to decreased emitting of the fluorescent signal through the skull, skin and fur of C57BL/6J mice, both in vivo and ex vivo analyses were conducted. Ex vivo, on day 5 there is no fluorescence enrichment measurable in the tumor region. The lesion seen in the fixed, coronal cut brain is a result of tumor implantation. The localization of the fluorescence detected in the right hemisphere on days 8 and 10 correlates with the tumor site in the fixed, coronal cut brain. Increased fluorescence intensity was detectable in a cryosection of a 20-day-old intracranially implanted tumor after i.v. injected 1D11-coupled AF594. Left, left hemisphere; right, right hemisphere; c, cerebellum. (c) Fluorescence lifetime of the free AF680 dye is higher than that of AF680 coupled 1D11 antibody measured 5 min after injection in a CD1-Foxn1nu mouse. After $24 \mathrm{~h}$ the free dye is almost eliminated from the body. The lifetime is almost reduced to that of the untreated mouse. Lifetime of the AF680 1D11 increases over time and is still detectable after $24 \mathrm{~h}$. The antibody-coupled fluorescence dye stays in the body. Fluorescence intensity and lifetime were measured by small animal fluorescence imaging. 

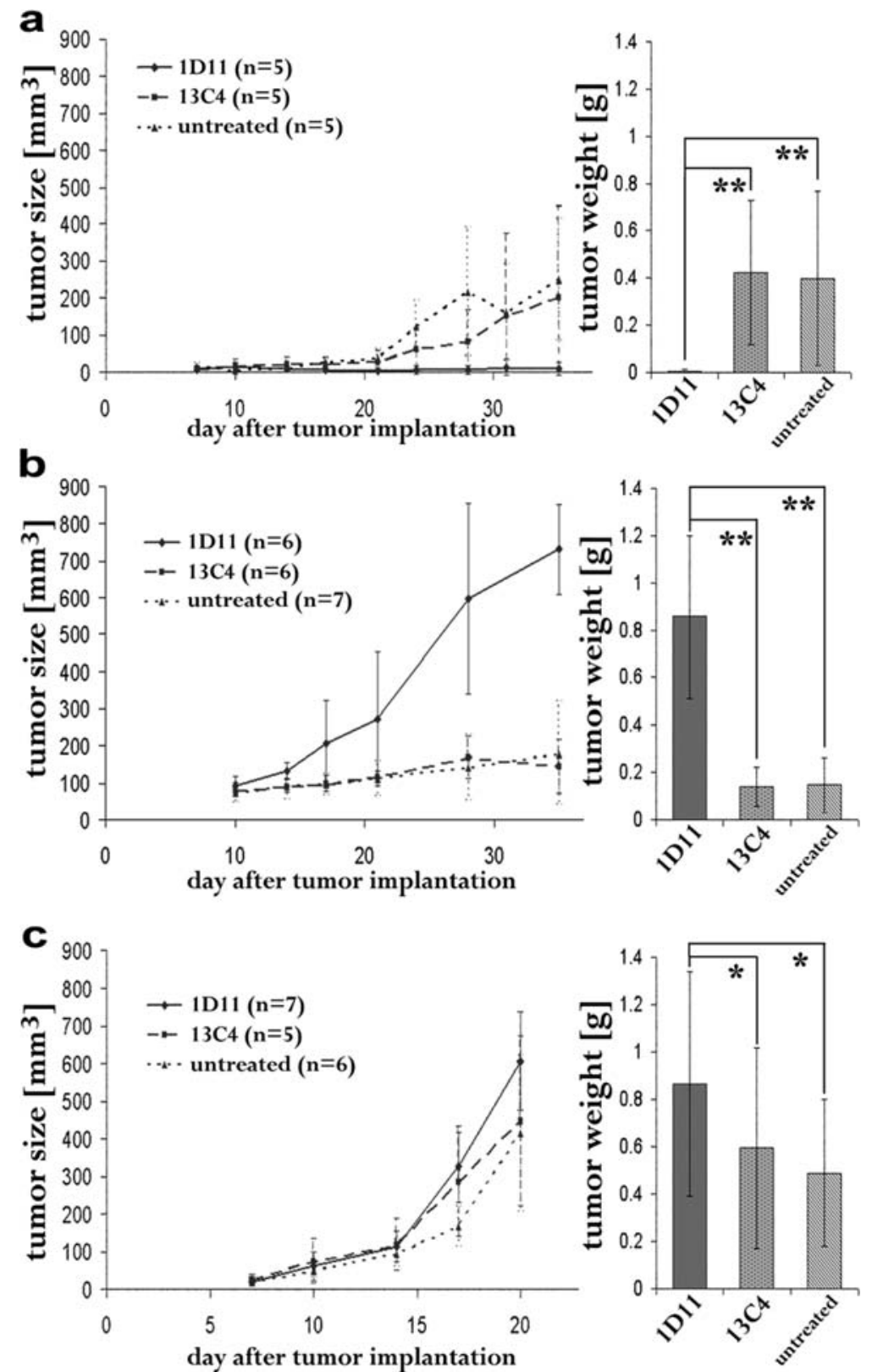

Figure 3. Tumor size of subcutaneously implanted gliomas. Left panels, tumor volumes; right panels, tumor weight at the end of the experiments. Treatment with 1D11, an anti-TGF- $\beta$ antibody, isotype $13 \mathrm{C} 4$ antibody or untreated controls. Tumors were measured with a caliper twice a week starting at day 8 after implantation. The size was estimated by the formula $V=4 / 3 \cdot \pi \cdot$ height $/ 2 \cdot$ length/2 $\cdot$ width/2. (a) Murine GL261 cells were implanted on the right flank of immunocompetent C57/BL/6J. (b) Human U87MG cells were implanted on the right flank of immunodeficient CD1-Foxn1nu mice. (c) Murine GL261 cells were implanted on the right flank of immuno-deficient CD1-Foxn1nu mice $\left({ }^{*} \mathrm{p}>0.073,{ }^{* *} \mathrm{p}<0.001\right)$.

growth inhibition and the weight of 1D11-treated tumors was significantly reduced at the end of the experiment compared to untreated tumors or tumors treated with the isotype control antibody, 13C4 (t-test: p<0.001; Fig. 3a). Three days after starting treatment the tumors of the 1D11 group did not differ in size compared to control tumors. Thereafter, 1D11 did not only inhibit the growth of GL261 gliomas but also reduced tumor size.

SC glioma treatment with $1 D 11$ leads to increased tumor size in immunocompromised mice. Treatment with 1D11 had contradictory effects on SC U87MG tumors in CD1-Foxn1nu mice. Mice treated with 1D11 showed increased growth rates and higher end weights compared with controls (t-test: $\mathrm{p}<0.001$; Fig. 3b). To verify whether the difference in growth behavior was caused by the tumor cell line itself, GL261 cells were subcutaneously implanted into CD1Foxn1nu mice and tumor-bearing mice were treated with 1D11. Not surprisingly, the GL261 tumors grew much faster in these immunocompromised mice. At day 21 after implantation, the tumors were already as big as at day 35 in the other models. As observed with the U87MG xenografts, treatment with 1D11 resulted in accelerated growth of these GL261 SC tumors (Fig. 3c), data that are in contrast to the inhibition of growth of the GL261 tumors that was observed in immunocompetent C57B1/6J mice treated with 1D11. 
a

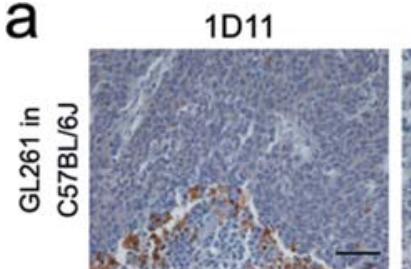

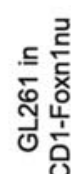

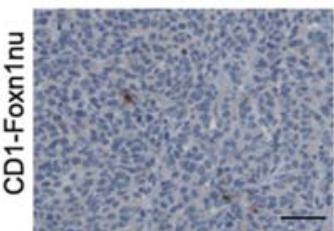

b

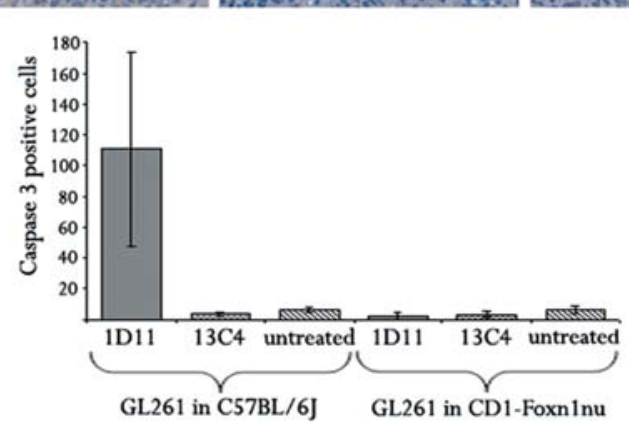

untreated
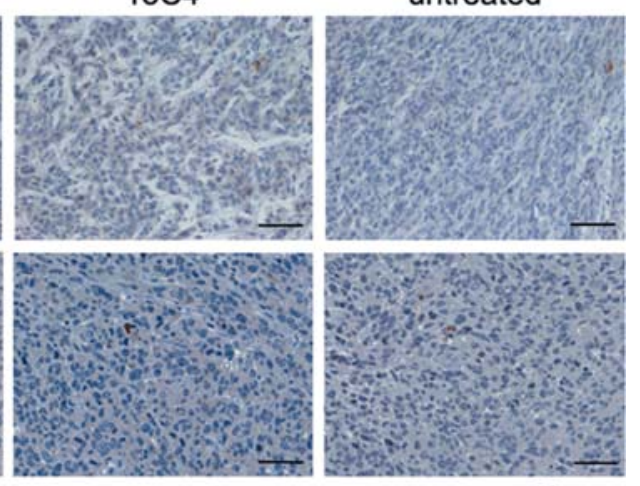

$-50 \mu \mathrm{m}$

Figure 4. Apoptotic cells in subcutaneous GL261 tumors of C57BL/6J and CD1-Foxn1nu mice. (a) Staining of paraffin sections for caspase-3 antibody. Caspase-3-positive cells are brown-black; nuclei are counterstained with hematoxylin. (b) Number of apoptotic cells in SC GL261 tumors. In the tumors grown in CD1-Foxn1nu mice there is no statistical difference in the number of apoptotic cells between the 1D11-treated and the control groups. In the tumors grown in C57BL/6J mice there is a statistically significant difference in the number of apoptotic cells between the 1D11-treated and the control groups. $\mathrm{n}=5$ each, $\mathrm{n}=2$ for the group GL261 in C57BL/6J mice treated with 1D11 (complete tumor remission in the other mice of the group).
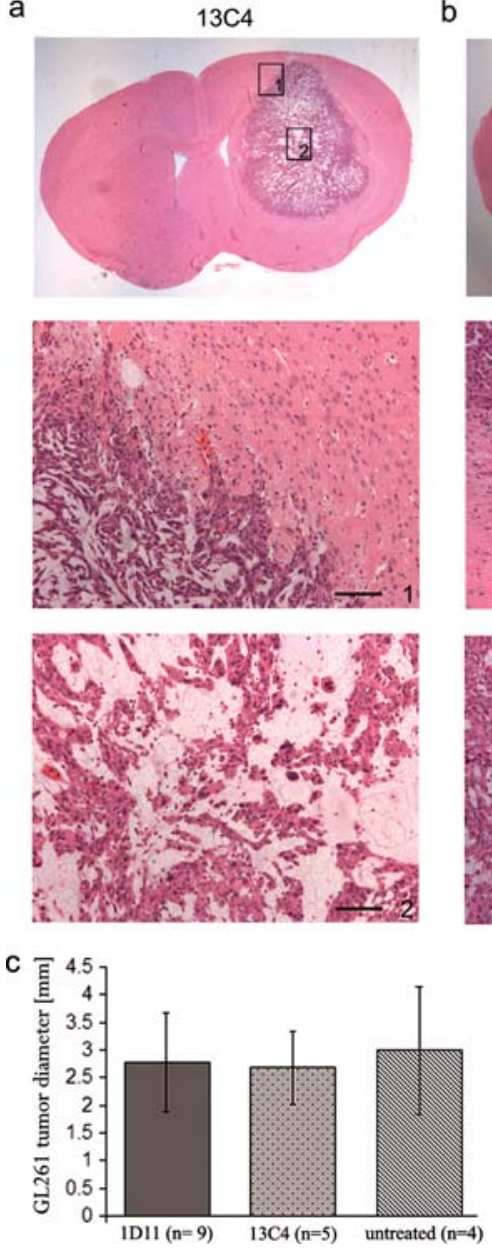

b
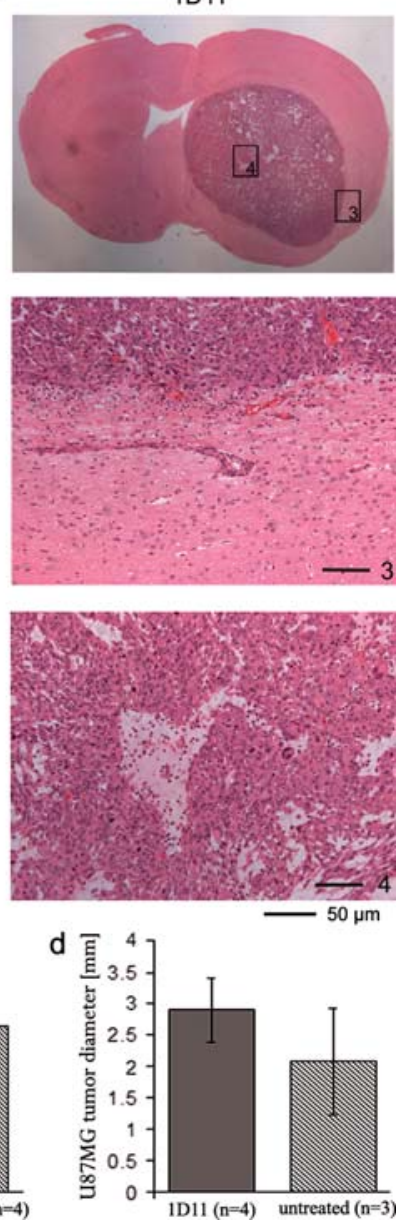

Immunohistochemical staining of U87 MG tumor sections was performed to determine the effects of 1D11 treatment on the tumor microenvironment. No differences were found regarding the number of Ki-67-positive proliferating tumor cells and MAC-3-positive macrophages after 1D11 treatment compared to the controls (data not shown). In addition, immunofluorescence microscopy for von Willebrand factor demonstrated that no changes were observed in microvessel density (data not shown). In not completely regressed SC GL261 tumors of 1D11-treated C57BL/6J mice increased amount of apoptotic cells was observed compared to 13C4treated and untreated control, although no such increase in apoptosis was observed in 1D11-treated GL261 and U87MG tumors from CD1-Foxn1nu mice (Fig. 4).

Morphology of orthotopic glioma in immunocompetent mice changes after treatment with 1D11. Given the differential effects of 1D11 on SC tumors depending on the immune status of the mice, and in order to minimize any artifactual effects of implanting glioma cells in a SC setting, an orthotopic

Figure 5. Overview and magnification of the H\&E-stained brain containing a 25-day intracranial GL261 tumor of a control (a) and a 1D11-treated (b) C57BL/6J mouse. (a) The tumor has an infiltration zone in which the tumor cells migrate into the healthy brain tissue (panel 1). The cells lie loosely in a matrix rich center of the tumor (panel 2). (b) Only few tumor cells infiltrate into the healthy tissue (here migration along a vessel) (panel 3). The cells in the tumor center show less stroma and matrix deposition. Necrosis is visible in the center (panel 4). (c and d) Mean maximum diameters of intracranial 1D11treated and control tumors measured from H\&E stained slices $(\mathrm{p}=0.491)$. 
model of glioma was incorporated into these studies to better mimic the clinical disease. Intracranial injection of GL261 cells into the brains of C57BL/6J mice or U87MG cells into the brains of CD1-Foxn1nu mice were conducted using stereotactic surgery into the parenchyma of the right hemisphere, and tumor-bearing mice were treated with 1D11 or controls. In immunodeficient mice the intracranial tumors treated with 1D11 tend to grow faster than the controls, as they did in the SC model (Fig. 5d). In contrast to the data from the SC tumor model, in immunocompetent $\mathrm{C} 57 \mathrm{BL} / 6 \mathrm{~J}$ mice the size of the intracranial tumors was not reduced by $1 \mathrm{D} 11$ antibody treatment compared to control gliomas. Histological evaluations revealed that mean tumor diameter measured at the tumor implantation site was equal between 1D11-treated mice and controls (Fig. 5c). However, 1D11 did exert pharmacodynamic effects on the tumors. Those treated with 1D11 contained less stroma and matrix deposition and resulted in a reduced infiltration and invasion of the tumor cells into the surrounding normal brain tissue (Fig. 5a and b).

\section{Discussion}

Although the growth of SC GL261 tumors was inhibited by $1 \mathrm{D} 11$ in immunocompetent, syngeneic C57B1/6 mice, the GL261 tumors grew faster in immunocompromised CD1-Foxn1nu mice treated with 1D11. This growth-promoting effect of 1D11 was also seen in experiments with SC U87MG tumors grown in CD1-Foxn1nu mice. The differences in effects based on the immune status of mice suggest that TGF- $\beta$ may be functioning differently during tumor growth in these different settings. In a study of Pan and colleagues (27) TGF-ß1-overexpressing cells formed smaller tumors in immunodeficient rats compared to tumors that developed from wild-type U87MG cells. In the present study, 1D11treated tumors, where, hypothetically, the amount of active TGF- $\beta$ is reduced, grew bigger. In both cases decreased TGF- $\beta$ led to increased tumor growth suggesting that TGF- $\beta$ may be suppressing the growth of SC tumors in an immunodeficient setting and may be exerting different effects in the tumor microenvironment depending on the status of the immune response to tumors.

For both immunocompetent and immunodeficient mice there were no histological differences between SC GL261 tumors of 1D11-treated animals and control animals based on H\&E staining (data not shown). In the immunodeficient setting, the proliferation of the tumor cells was not altered in response to $1 \mathrm{D} 11$ treatment nor was there a change in the number of tumor associated macrophages. In immunodeficient mice there was no difference in the number of apoptotic cells in tumors treated with 1D11 compared to tumors treated with controls, and the vessel density was also not affected by 1D11 treatment. Similar results were also obtained by Pan and colleagues who found no difference in angiogenesis, apoptosis or the number of tumor-infiltrating lymphocytes in tumors resulting from the two cell lines when injected into immunodeficient rats (27). Although no changes in microvessel density were observed in this study or published data, results from Dohgu et al (28) and Walshe et al (29) indicate that the functionality of vessels is affected by TGF- $\beta$. TGF- $\beta$ can upregulate expression of VEGF which induces angiogenesis and levels of VEGF can affect normalization of blood vessels. Neutralization of TGF- $\beta$ leading to decreased VEGF may modify the functionality in glioma-associated vessels. Thus, the more normalized blood supply in 1D11-treated tumors could result in faster growth, however, experiments would need to be conducted to confirm or refute this hypothesis.

This hypothesis would not explain why inhibition of GL261 SC tumor growth was observed following treatment with $1 \mathrm{D} 11$ in the immunocompetent setting. 1D11 treatment of immunocompetent C57BL/6J mice leads to growth inhibition or total regression of pre-established SC GL261 tumors. TGF- $\beta$ is a potent immunosuppressor, and the effects of neutralizing TGF- $\beta$ on immune effector function would not be evident in an immunocompromised setting. The growth stimulating effect of 1D11 antibody treatment of GL261 tumors seen in CD1-Foxn1nu mice may be overwhelmed by the enhancement of the immune system with a net effect of not only growth reduction but also complete remission of the pre-established tumor. There was an increase in the number of apoptotic cells in the two existing small tumors of 1D11treated C57BL/6J mice compared to controls, and a similar increase in apoptosis was not detectable in tumors growing in the CD1-Foxn1nu mice. TGF- $\beta$ inhibits proliferation and activity of cytotoxic T cells and NK cells $(16,17)$, which can induce apoptosis in tumor cells. TGF- $\beta$ binding soluble TGF- $\beta$ receptors block the immunosuppressive effect of TGF- $\beta$ on NK cells (30). In an intracranial GL261 model testing 1D11 in combination with a glioma-associated antigen vaccine, the amount of CD-3+ $\mathrm{T}$ cells in the tumor is not affected by the $1 \mathrm{D} 11$ as a single agent or in combination with the vaccine (22). However, TGF- $\beta$ inhibition by $1 \mathrm{D} 11$ caused an increase in $\mathrm{CD}^{+} \mathrm{T}$ cells and a decrease in $\mathrm{T}$ regs in the brain tumors; an effect that was enhanced by the vaccine (22). However, for the tumor size-reducing effect of 1D11 treatment in intracranially growing gliomas the immunity against the glioma cells has to be enhanced by vaccination (22). Therefore, analysis of the effects of 1D11 on these immune effector cells is warranted.

Interestingly, treatment of intracranial GL261 tumors with 1D11 antibody resulted in neither an increase nor a decrease in glioma growth in $\mathrm{C} 57 \mathrm{BL} / 6 \mathrm{~J}$ mice. These data, in contrast to that from the SC model, suggest that the local tumor microenvironment may be playing a role in the efficacy of 1D11 against these gliomas. In contrast to the current data, Uhl et al (9) found a growth reduction of intracranially implanted SMA 560 gliomas in an immunocompetent mouse model after oral treatment of the mice with SD-208, a small molecule TGF- $\beta$ receptor II inhibitor. In addition to potential differences in the models used in these studies, the small molecule would, hypothetically, have better tumor penetration than the larger antibody and could account for efficacy observed with SD-208 as opposed to 1D11.

It is also possible that the blood-brain/blood-tumor barrier may be limiting the bioavailability of the antibody to the tumor, and to address this, tumor localization studies were conducted incorporating imaging with fluorescently-labeled 1D11. Studies were conducted with both the intracranial model as well as the SC model to see if differences in observed effects with $1 \mathrm{D} 11$ can be attributed to altered tumor localization of the antibody. 
The labeled 1D11 antibody is detectable in the SC U87MG tumor of CD1-Foxn1nu mice as well as in the SC GL261 tumor of C57/BL/6J. 1D11 entered the tumor tissue and was detectable at vessel walls as well as in the tumor tissue. Measurement of fluorescence intensity in mice during the course of therapy showed that high concentrations of the antibody were found in the SC gliomas compared to the remaining body (observed in CD1-Foxn1nu mice) and that 1D11 does not completely disappear from the tumor during the treatment period (observed in CD1-Foxn1nu as well as in C57/BL/6J mice). Due to the fur the measured fluorescence intensities cannot be compared between C57/BL/6J and CD1Foxn1nu mice. In vivo imaging of the intracranial tumors in C57BL/6J mice after intravenous injection of AF680-1D11 was problematic due to impedance of a light signal by the implantation lesion and the regrowth of the black fur of mice. In ex vivo measurements, however, the excised brains showed increased fluorescence intensity in the tumor region. Importantly, fluorescently-labeled 1D11 was observed in the glioma tissue, but not in surrounding normal brain parenchyma. This effect could be due to 1D11 escaping through the leaky vasculature associated with gliomas, while the antibody can not cross the intact blood-brain barrier in normal brain tissue. These data confirm that 1D11 can localize to both SC as well as intracranial tumors.

However, the degree of accumulation of neutralizing antibody to the tumors may be important to observing efficacy against these orthotopic gliomas. In the SC tumors the AF680 1D11 was detectable in the tumor from the beginning of the therapy on day 5. In the orthotopic model the treatment started on day three after tumor cell implantation. This was done, because the tumor is lethal in a shorter time frame than in the SC model. At this time-point the AF680 1D11 fluorescence was not detected in the brain tumor after i.v. administration. In fact, day 8 after tumor implantation (five days following the first therapeutic injection in the therapy) was the first day in which 1D11 could be detected in these brain tumors. In this aggressive glioma model, perhaps the accumulation of 1D11 does not reach a critical threshold concentration in the tumors until the tumor has already advanced to an aggressive state. In a study of Hau et al (31) TGF-B2-specific antisense phosphorothioate oligonucleotide AP 12009, which has been shown to reduce the amount of released TGF- $\beta$ in primary glioma cell lines, was tested in humans with malignant gliomas and anaplastic astrocytomas (31). In this study, patients with the smallest tumor residue after resection showed the best response to the local administrated therapy. The patients did not respond to the treatment immediately, but after a minimum of 28 days, some of the patients showed stable disease. In one case tumor progression was observed before the patient had a complete remission. Unfortunately, given the aggressiveness of this model, the tumors in the intracranial model used in the current studies may be growing too rapidly in the confined space of the brain to observe any inhibition of tumor growth by 1D11.

Although no efficacy was observed with 1D11 in regards to reduction of intracranial tumor growth, neutralization of TGF- $\beta$ had pharmacodynamic effects on these orthotopic gliomas. The tumors of 1D11-treated mice were more marginalized than the control tumors and the invasiveness of tumor cells into the healthy brain tissue was reduced. There was a significant reduction in the number of interdigitations into the surrounding normal brain tissue in mice treated with 1D11. This finding is consistent with the results of Uhl and colleagues (9) in glioma cell lines and with the results of Wesolowska et al (11) in primary glioma cells, where the inhibition of TGF- $\beta$ signaling reduced the motility of glioma cells. As discussed earlier, 1D11 also had no efficacy as a single agent in the GL261 model conducted by Ueda and colleagues, but did show effects on immune effector function and was able to enhance the efficacy of a vaccine against these gliomas. Neutralization of TGF- $\beta$ by 1 D11 appears to have anti-tumorigenic effects on these orthotopic gliomas, however, the aggressiveness of this model may not allow for these to manifest as a survival advantage or tumor growth inhibition.

Taken together these data show that the pan-neutralizing anti-TGF- 3 antibody, 1D11, can localize to both SC and intracranial gliomas. The effects that this neutralizing antibody may have on tumor growth and progression appears to be dependent on the status of the immune system as well as the location of the tumor itself, suggesting that the role of TGF- $\beta$ in tumor growth is context-dependent. Given the differences in response to $1 \mathrm{D} 11$ in this study, strategies targeting TGF- $\beta$ in the clinic should also be context-dependent.

\section{Acknowledgments}

We thank Professor S. Van Gool from the University Hospital of Leuven, Leuven, Belgium for providing the GL261 cell line. Many thanks to Regina Krügener and Rita Spohr-Müller for their excellent technical assistance. Genzyme Corporation supported the work by a grant.

\section{References}

1. Stupp R, Hegi ME, van den Bent MJ, Mason WP, Weller M, Mirimanoff RO and Cairncross JG: Changing paradigms - an update on the multidisciplinary management of malignant glioma. Oncologist 11: 165-180, 2006.

2. Hutterer M, Gunsilius E and Stockhammer G: Molecular therapies for malignant glioma. Wien Med Wochenschr 156: 351-363, 2006.

3. Sathornsumetee S and Rich JN: Designer therapies for glioblastoma multiforme. Ann NY Acad Sci 1142: 108-132, 2008.

4. Baselga J: The EGFR as a target for anticancer therapy - focus on cetuximab. Eur J Cancer 37: 16-22, 2001.

5. Wen PY, Yung WK, Lamborn KR, Dahia PL, Wang Y, Peng B, Abrey LE, Raizer J, Cloughesy TF, Fink K, Gilbert M, Chang S, Junck L, Schiff D, Lieberman F, Fine HA, Mehta M, Robins HI, De Angelis LM, Groves MD, Puduvalli VK, Levin V, Conrad C, Maher EA, Aldape K, Hayes M, Letvak L, Egorin MJ, Capdeville R, Kaplan R, Murgo AJ, Stiles C and Prados MD: Phase I/II study of imatinib mesylate for recurrent malignant gliomas: North American Brain Tumor Consortium Study 99-08. Clin Cancer Res 12: 4899-4907, 2006.

6. Cloughesy TF, Kuhn J, Robins HI, Abrey L, Wen P, Fink K, Lieberman FS, Mehta M, Chang S, Yung A, De Angelis L, Schiff D, Junck L, Groves M, Paquette S, Wright J, Lamborn K, Sebti SM and Prados M: Phase I trial of tipifarnib in patients with recurrent malignant glioma taking enzyme-inducing antiepileptic drugs: a North American Brain Tumor Consortium Study. J Clin Oncol 23: 6647-6656, 2005.

7. Ermoian RP, Kaprealian T, Lamborn KR, Yang X, Jelluma N, Arvold ND, Zeidman R, Berger MS, Stokoe D and HaasKogan DA: Signal transduction molecules in gliomas of all grades. J Neurooncol 91: 19-26, 2009.

8. Brandes AA, Franceschi E, Tosoni A, Hegi ME and Stupp R: Epidermal growth factor receptor inhibitors in neuro-oncology: hopes and disappointments. Clin Cancer Res 14: 957-960, 2008. 
9. Uhl M, Aulwurm S, Wischhusen J, Weiler M, Ma JY, Almirez R, Mangadu R, Liu Y-W, Platten M, Herrlinger U, Murphy A, Wong DH, Wick W, Higgins LS and Weller M: SD-208, a novel transforming growth factor $\beta$ receptor I kinase inhibitor, inhibits growth and invasiveness and enhances immunogenicity of murine and human glioma cells in vitro and in vivo. Cancer Res 64: 7954-7961, 2004

10. Baumann F, Leukel P, Doerfelt A, Beier CP, Dettmer K, Oefner PJ, Kastenberger M, Kreutz M, Nickl-Jockschat T, Bogdahn U, Bosserhoff AK and Hau P: Lactate promotes glioma migration by TGF-B2-dependent regulation of matrix metalloproteinase-2. Neurooncology 11: 368-380, 2009.

11. Wesolowska A, Kwiatkowska A, Slomnicki L, Dembinski M, Master A, Sliwa M, Franciszkiewicz K, Chouaib S and Kaminska B: Microglia-derived TGF- $\beta$ as an important regulator of glioblastoma invasion - an inhibition of TGF- $\beta$ dependent effects by shRNA against human TGF- $\beta$ type II receptor. Oncogene 27: 918-930, 2008.

12. Akhurst RJ and Derynck R: TGF-beta signaling in cancer - a double-edged sword. Trends Cell Biol 11: 44-51,2001.

13. Pepper MS: Transforming growth factor-beta: vasculogenesis, angiogenesis, and vessel wall integrity. Cytokine Growth Factor Rev 8: 21-43, 1997.

14. Koochekpour S, Merzak A and Pilkington GJ: Vascular endothelial growth factor production is stimulated by gangliosites and TGF-beta isoforms in human glioma cells in vitro. Cancer Lett 102: 209-215, 1996.

15. Friese MA, Steinle A and Weller M: The innate immune response in the central nervous system and its role in glioma immune surveillance. Onkologie 27: 487-491, 2004.

16. Weller M and Fontana A: The failure of current immunotherapy for malignant glioma. Tumor-derived TGF-beta, T-cell apoptosis, and the immune privilege of the brain. Brain Res Brain Res Rev 21: 128-151, 1995.

17. Bodmer S, Strommer K, Frei K, Spiel C, De Tribolet N, Heid I and Fontana A: Immunosuppression and transforming growth factor-beta in glioblastoma. Preferential production of transforming growth factor-beta 2. J Immunol 143: 3222-3229, 1989.

18. Ahmadzadeh $\mathrm{M}$ and Rosenberg SA: TGF-beta 1 attenuates the acquisition and expression of effector function by tumor antigenspecific human memory CD8 T cells. J Immunol 174: 5215-5223, 2005.

19. Platten M, Wick W and Weller M: Malignant glioma biology: role of TGF-beta in growth, motility, angiogenesis, and immune escape. Microsc Res Tech 52: 401-410, 2001.

20. Liu Y, Wang Q, Kleinschmidt-DeMasters BK, Franzusoff A, $\mathrm{Ng} \mathrm{K-Y}$ and Lillehei KO: TGF-B2 inhibition augments the effect of tumor vaccine and improves the survival of animals with pre-established brain tumors. J Neurooncol 81: 149-162, 2007.

21. Nam JS, Terabe M, Mamura M, Kang MJ, Chae H, Stuelten C, Kohn E, Tang B, Sabzevari H, Anver MR, Lawrence S, Danielpour D, Lonning S, Berzofsky JA and Wakefield LM: An anti-transforming growth factor beta antibody suppresses metastasis via cooperative effects on multiple cell compartments. Cancer Res 68: 3835-3843, 2008.
22. Ueda R, Fujita M, Zhu X, Sasaki K, Kastenhuber ER, Kohanbash G, McDonald HA, Harper J, Lonning S and Okada H: Systemic inhibition of transforming growth factorbeta in glioma-bearing mice improves the therapeutic efficacy of glioma-associated antigen peptide vaccines. Clin Cancer Res 15: 6551-6559, 2009.

23. Dasch JR, Pace DR, Waegell W, Inenaga D and Ellingsworth L: Monoclonal antibodies recognizing transforming growth factor- $\beta$, bioactivity neutralization and transforming growth factor 82 affinity purification. J Immunol 142: 1536-1541, 1998.

24. Keren S, Gheysens O, Levin CS and Gambhir SS: A comparison between a time domain and continuous wave small animal optical imaging system. IEEE Trans Med Imaging 27: 58-63, 2008.

25. Kumar AT, Raymond SB, Dunn AK, Bacskai BJ and Boas DA: A time domain fluorescence tomography system for small animal Imaging. IEEE Trans Med Imaging 27: 1152-1163, 2008.

26. McCormack E, Micklem DR, Pindard LE, Silden E, Gallant P, Belenkov A, Lorens JB and Gjertsen BT: In vivo optical imaging of acute myeloid leukemia by green fluorescent protein: timedomain autofluorescence decoupling, fluorophore quantification, and localization. Mol Imaging 6: 193-204, 2007.

27. Pan JJ, Chang WJ, Barone TA, Plunkett RJ, Ostrow PT and Greenberg SJ: Increased expression of TGF-beta1 reduces tumor growth of human U-87 glioblastoma cells in vivo. Cancer Immunol Immunother 55: 918-927, 2006.

28. Dohgu S, Yamauchi A, Takata F, Naito M, Tsuruo T, Higuchi S, Sawada Y and Kataoka Y: Transforming growth factor-beta1 upregulates the tight junction and P-glycoprotein of brain microvascular endothelial cells. Cell Mol Neurobiol 24: 491-497, 2004.

29. Walshe TE, Saint-Geniez M, Maharaj AS, Sekiyama E, Maldonado AE and D'Amore PA: TGF-beta is required for vascular barrier function, endothelial survival and homeostasis of the adult microvasculature. PLoS One 4: e5149, 2009.

30. Naumann U, Maass P, Gleske AK, Aulwurm S, Weller M and Eisele G: Glioma gene therapy with soluble transforming growth factor-beta receptors II and III. Int J Oncol 33: 759-765, 2008 .

31. Hau P, Jachimczak P, Schlingensiepen R, Schulmeyer F, Jauch T, Steinbrecher A, Brawanski A, Proescholdt M, Schlaier J, Buchroithner J, Pichler J, Wurm G, Mehdorn M, Strege R, Schuierer G, Villarrubia V, Fellner F, Jansen O, Straube T, Nohria V, Goldbrunner M, Kunst M, Schmaus S, Stauder G, Bogdahn U and Schlingensiepen KH: Inhibition of TGF-beta2 with AP 12009 in recurrent malignant gliomas: from preclinical to phase I/II studies. Oligonucleotides 17: 201-212, 2007. 